\title{
Exploring radiation safety knowledge among nurses
}

\author{
๑D Berna Uçan, ĐHasibe Gökçe Çınar \\ Dr. Sami Ulus Training and Research Hospital, Department of Pediatric Radiology, Ankara, Turkey
}

Cite this article as: Uçan B, Çınar G. Exploring radiation safety knowledge among nurses. Anatolian Curr Med J 2022; 4(1); 55-58.

\begin{abstract}
Aim: As part of the medical team, nurses often take part in both controlled and supervised radiation fields. For this reason, nurses need to know about radiation and radiation safety. This study is aimed to determine the knowledge level of nurses in a tertiary pediatric hospital about radiation safety through a questionnaire.

Material and Method: Within the scope of the survey conducted, the age, gender and years of experience data of the participants were collected. In addition, they have been asked whether they have received radiation safety training throughout their professional life and whether they want to receive such training now. Basic questions about radiation safety and questions to measure the general level of information about radiation are also addressed.

Results: The link of the survey was delivered to 583 nurses in the study center and 313 nurses completed the questionnaire. $53.67 \%$ of the nurses stated that they do not know the name of the beam used for x-ray imaging. $64.28 \%$ of the nurses stated that they did not receive such education in their professional life. $78.57 \%$ of all participants stated that they wanted to receive a training how to protect themselves while X-ray is being performed in their environment.

Conclusion: A significant number of nurses do not receive radiation safety training. Those who received such an education state that they are not satisfied with the training. Nurses want to receive radiation safety training. Trainings with regular and appropriate content can increase nurses' radiation safety knowledge and awareness.
\end{abstract}

Keywords: Awareness nurse, knowledge, radiation, safety

\section{INTRODUCTION}

Radiation safety is a necessity that must be ensured not only for patients exposed to radiation but also for staff working in this environment. National and international institutions have already determined the limits of iatrogenic radiation exposure. However, it is likely to be beneficial for individuals working in ionizing radiation environments to know basic radiation safety and fundamental concepts of radioactivity both for their own health and compliance with daily radiation safety practices $(1,2)$.

In healthcare institutions, areas with a risk of exposure to doses of $1 \mathrm{mSv}$ radiation or more per year are considered radiation areas. Besides, radiation areas are divided into two as controlled and supervised areas. The risk of radiation exposure is higher, and dosimeters must be used in controlled areas. Although there is a higher risk of radiation exposure in supervised areas, the use of dosimeters is not mandatory $(3,4)$.
Nurses, as part of the healthcare staff, are frequently employed in both controlled and supervised areas (5). For example, nurses working in areas where portable radiographs are utilized intensively (e.g., intensive care units) have increased exposure risk $(6,7)$. Hence, nurses are among the professionals who should know about radiation and radiation safety. By the way, radiation knowledge plays a vital role in ensuring radiation safety $(4,8)$. For this reason, both nurses and other healthcare professionals frequently engage in radiation safety training $(6,7)$. The relevant literature hosts several studies measuring nurses' awareness of radiation and radiation safety. Yet, most of these studies found nurses' awareness of radiation to be insufficient $(6,8)$.

Ultimately, this study aimed to reveal the radiation and radiation safety knowledge of nurses working in a tertiary pediatric hospital through a questionnaire. In this way, the findings may help increase radiation safety awareness and pave the way for relevant training programs. 


\section{MATERIAL AND METHOD}

The study was carried out with the permission of Etlik Zübeyde Hanım Gynecology Training and Research Hospital Clinical Researh Ethics Committee (Date: 23.09.2020, Decision No: 2020/136). All procedures were carried out in accordance with the ethical rules and the principles of the Declaration of Helsinki.

The present study was prospective survey research carried out with nurses working in a tertiary pediatric hospital. We obtained ethical approval for the study from relevant bodies. Participation in the study was entirely voluntary, and we included an informed consent section at the beginning of the questionnaire that contains a summary of research purpose, states that the participants' personal information will not be collected, and emphasizes that the participation is on a voluntary basis.

Prior to the study, we performed a pilot study with 20 nurses of varying experience levels to reveal the suitability and comprehensibility of the questions in the survey. Then, we finalized the questionnaire based on the feedback.

Next, we transferred the questionnaire form to the online environment and delivered the relevant electronic link to the participants via a social media platform (WhatsApp). We compiled the responses without any personal information. Filling out the questionnaire more than once was prevented using the internet protocol (IP) address restriction provided by the online platform.

In the study, we collected demographic characteristics of the participants (age, sex, and years of experience). In addition, we inquired whether they had received radiation safety training during their formal education and professional life and whether they would like to receive such training now. Moreover, the questionnaire included basic questions about radiation safety and questions to measure the general radiation knowledge of the participants. Appendix 1 presents the questionnaire.

We analyzed the data using the Statistical Package for Social Sciences (SPSS), Windows 20 (IBM SPSS Inc., Chicago, IL). We referred to the Kolmogorov-Smirnov test to check the normality of data distribution and showed normally distributed data as mean and standard deviation. The distribution of responses was shown as percentages. Finally, we performed the Mann-Whitney $\mathrm{U}$ test, student $\mathrm{t}$ test and Chi-Square test to compare the responses by age, professional experience, and sex.

A p-value of $<0.05$ was considered statistically significant in all analyses.

\section{RESULTS}

We delivered the questionnaire link to 583 nurses employed in the center where we conducted the study and got responses from a total of 313 nurses. Among them, $245(78.2 \%)$ were females, and $68(21.7 \%)$ were males. The mean age of the participants was $37.3 \pm 11.5$, while they had an average of $17.2 \pm 6.8$ years of work experience.

The survey includes two questions to measure the participants' general radiation knowledge. The results revealed that $53.67 \%$ of the nurses did not know the name of the beam used for X-ray imaging. Only $17.89 \%$ were able to give an "X-ray" response (Table 1). About half of the participants (42.85\%) stated knowing about natural radiation.

\begin{tabular}{|lc|}
\hline \multicolumn{2}{|l|}{ Table 1. What is the name of the rays used in X-ray research? } \\
\hline Answer & Number (\%) \\
\hline Alfa & $67(21.4 \%)$ \\
X & $56(17.89 \%)$ \\
Gama & $22(7.02 \%)$ \\
I don't Know & $168(53.67 \%)$ \\
\hline
\end{tabular}

More than half of the participants (57.14\%) reported being knowledgeable about what they should do to protect themselves while performing an X-ray examination; however, only $31.19 \%$ were able to accurately know the minimum distance (1 meter) from an X-ray generator (Table 2).

Table 2. The answers given to the questions about what is the distance required to be protected from X-rays in the environment.

Answer Number (\%)

1 meter $100(31.19 \%)$

3 meter $\quad 190(60.70 \%)$

5 meter $22(7.02 \%)$

Correct answer is shown in bold (Hellwig BJ, Wilson B. Quality Improvement Related to Radiation Safety of Chest Radiography in the NICU. Radiology management. 2013;35(2).)

We found the rates of those not receiving any training during their education and professional life on how to protect themselves during an X-ray examination to be $46.42 \%$ and $64.28 \%$, respectively.

More than half of the participants (56.2\%) received training on radiation protection during their education or professional life. Yet, among them, the majority $(71.25 \%)$ did not consider the training sufficient. Besides, $78.57 \%$ of all participants desired to receive training covering what they need to do to protect themselves during an $\mathrm{X}$-ray examination.

We could not find any significant differences between the responses by sex $(\mathrm{p}=0.07)$. 
We found an inverse and significant relationship between age and knowledge of radiation protection. We divided the participants into two groups, accepting the threshold of 35 years, and compared the responses between the groups. Similarly, we formed two groups by professional experience, as those with less than 15 years of experience and the others, and compared their responses. Findings showed that those below 35 years and with less than 15 years of professional experience gave a significantly higher response of "Yes" to the question "Do you have any information about the methods of protecting yourself when you are needed for an X-ray examination?" $(p=0.03)$. In addition, a significantly larger percentage of these participants gave a correct answer to the question about the minimum distance (1 meter) from an X-ray generator during an examination $(\mathrm{p}=0.04)$. Again, those below 35 years and with less than 15 years of professional experience received radiation protection training during their formal education significantly higher than the others $(\mathrm{p}=0.001)$. However, the other responses/paramethers (except the knowledge of radiation) did not significantly differ by age and professional experience.

\section{DISCUSSION}

Nurses constitute a remarkable part of healthcare personnel and are widely employed in all types of units in hospitals, which may make them vulnerable to occupational radiation exposure. In the literature, a significant number of nurses do not know how to protect themselves from ionizing radiation during their professional life. Besides, most nurses consider their previous education on radiation protection to be insufficient and are willing to receive more comprehensive education on this subject $(10,11)$.

Natural radiation comes out from natural sources, especially from radon gas. Apart from radon gas, one may be exposed to natural radiation through gamma rays, cosmic rays, food, drinks, and air. Artificial radiation, on the other hand, occurs as a result of X-rays and artificial radioactive materials used for medical, agricultural, and industrial purposes. Of these, medical applications account for more than $95 \%$ of artificial radiation. The annual dose for artificial radiation is on average $0.3 \mathrm{mSv}$ $(12,13)$.

Radiation caused by medical interventions stands as a problem that should be emphasized for both patients and healthcare personnel, and its effects should be minimized. At this point, the most important and indispensable step of radiation safety is education $(14,15)$.

Recent years have witnessed a plethora of studies aiming to measure healthcare workers' radiation and radiation safety knowledge to design appropriate training programs and increase awareness of radiation safety $(16,17)$. Nurses are also one of the target groups of such studies. In their research, Morishima et al. (17) concluded that $58.2 \%$ of the nurses did not know about the types of radiation. Our data overlap the finding in the literature; only a few of our participants were able to specify the name of the ionizing beam used in the X-ray examination as "X-ray." Less than half of the participants knew about natural radiation. Overall, we may assert that the participating nurses have a lack fundamental radiation knowledge $(18,19)$.

Although more than half of the participants knew about protecting themselves from radiation, only about $1 / 3$ knew correctly the minimum distance required to set from an X-ray generator. This situation can be considered an indirect indicator of the lack of knowledge, even for those thinking to have sufficient knowledge about the topic. Similarly, several studies in the literature $(6,14)$ concluded that most nurses do not know the safe distance to stand from an X-ray generator during an examination.

The literature also emphasizes that nurses are not given adequate radiation safety training, and their level of knowledge often remains poorer than that of physicians and radiologic technologists $(10,20)$. Similarly, we found a significant part of the participants did not receive radiation safety training during their education or professional life, and the majority of those receiving such training was not satisfied with the training. In addition, $78.57 \%$ of the nurses desired to receive radiation safety training. Similar to our data, Morishima et al. (17) found $85.6 \%$ of the nurses to report that they would like to attend periodic radiation safety seminars.

In their study, Kim et al. (10) showed that nurses over 40 comply more with radiation safety precautions. Partly different from the literature, our data demonstrated that young nurses had more radiation and radiation safety knowledge than experienced nurses. In addition, the rate of receiving radiation safety training during formal education was found to be high among young nurses, which may refer to that radiation safety training has started to take place more in the curriculum of healthcare professionals in the last two decades. However, in parallel with the literature, both young and experienced nurses tended to consider the relevant training to be insufficient. Overall, it is not prudent to assert there is a need for radiation safety training for nurses of all age groups.

There are some limitations of our study. First, since we carried out the study in a single center, the findings may only present a preliminary situation about the subject. Therefore, multicenter studies with larger samples may be more reliable. Second, the study was carried only with nurses and did not make a comparison with physicians 
or other auxiliary healthcare personnel. If we had carried out the study immediately after any radiation safety training, we could have made a robust evaluation of the effectiveness of the training.

\section{CONCLUSION}

To conclude, nurses, an essential part of the healthcare staff and often exposed to in-hospital radiation, do not have sufficient knowledge of general radiation and radiation safety. A significant portion of the nurses did not receive radiation safety training, and those who did are not satisfied with the training. Overall, nurses want to receive radiation safety training; therefore, regular and appropriate training may increase nurses' knowledge and awareness of radiation safety.

\section{ETHICAL DECLARATION}

Ethics Committee Approval: The study was carried out with the permission of Etlik Zübeyde Hanım Gynecology Training and Research Hospital Clinical Researh Ethics Committee (Date: 23.09.2020, Decision No: 2020/136).

Informed Consent: All patients signed the free and informed consent form.

Referee Evaluation Process: Externally peer-reviewed.

Conflict of Interest Statement: The authors have no conflicts of interest to declare.

Financial Disclosure: The authors declared that this study has received no financial support.

Author Contributions: All of the authors declare that they have all participated in the design, execution, and analysis of the paper, and that they have approved the final version.

\section{REFERENCES}

1. Gökharman DF, Aydın S, Koşar PN. Radyasyon güvenliğinde mesleki olarak bilmemiz gerekenler. SDÜ Sağlık Bilimleri Derg 2016; 7: 35-40.

2. Frane N, Bitterman A. Radiation Safety and Protection 2021 May 29. In: StatPearls [Internet]. Treasure Island (FL): StatPearls Publishing; 2021.

3. Organization WH. International basic safety standards for protection against ionizing radiation and for the safety of radiation sources. 1994.

4. International Commission on Radiological Protection. The 2007 Recommendations of the International Commission on Radiological Protection. ICRP Publication 103. Annals of the ICRP 2007; 37: 2.

5. Cupitt J M, Vinayagam S, McConachie I. Radiation exposure of nurses on an intensive care unit. Anaesthesia 2001; 56: 183.

6. Dianati M, Zaheri A, Talari HR, Deris F, Rezaei S. Intensive care nurses' knowledge of radiation safety and their behaviors towards portable radiological examinations. Nursing Midwifery Studies 2014; 3: e23354.
7. Kang SG, Lee EN. Knowledge of radiation protection and the recognition and performance of radiation protection behavior among perioperative nurses. J Muscle Joint Health 2013; 20: 24757.

8. World Health Organization. Global Initiative on Radiation Safety in Healthcare Settings. Technical Meeting Report. 2008.

9. Amis Jr ES, Butler PF, Applegate KE, et al. American College of Radiology white paper on radiation dose in medicine. J Am College Radiol 2007 ; 4: 272-84

10. Kim O, Kim MS, Jang HJ, et al. Radiation safety education and compliance with safety procedures-The Korea Nurses' Health Study. J Clin Nurs 2018; 27 : 2650-60.

11. Ohno K, Kaori T. Effective education in radiation safety for nurses. Radiation Protection Dosimetry 2011; 147: 343-5.

12. Szarmach A, Piskunowicz M, Świętoń D, et al. Radiation safety awareness among medical staff. Polish J Radiol 2015; 80: 57.

13. International Commission on Radiological Protection. Education and training in radiological protection for diagnostic and interventional procedures. ICRP Publication 113. Annals of the ICRP 2009; 39: 5.

14. Sheyn DD, Racadio JM, Ying J, Patel MN, Racadio JM, Johnson ND. Efficacy of a radiation safety education initiative in reducing radiation exposure in the pediatric IR suite. Pediatr Radiol 2008; 38: 669-74.

15. Choi GN, Jeon JS, Kim YW. Radiation exposure dose on persons engaged in radiation-related industries. J Korean Soc Radiol 2002; 6: 27-37.

16. Yoon SJ, Oh JN, Im MH. Knowledge, attitude, and education needs of radiation protection among nursing students and nurses. J Korea Contents Assoc 2016; 16: 563-72.

17. Morishima Y, Chida K, Katahira Y, Seto H, Chiba H, Tabayashi K. Need for radiation safety education for interventional cardiology staff, especially nurses. Acta Cardiologica 2016; 71: 151-5.

18. Alotaibi M, Saeed R. Radiology nurses' awareness of radiation. J Radiol Nurs 2005; 25: 7-12.

19. 19.Rassin M, Granat P, Berger M, Silner D. Attitude and knowledge of physicians and nurses about ionizing radiation. J. Radiol. Nurs 2005; 24: 26-30.

20. Badawy MK, Mong KS, Lykhun UP, Deb P. An assessment of nursing staffs' knowledge of radiation protection and practice. J Radiol Prot 2016; 36: 178-83. 